\title{
Simulações do perfil vertical do vento na camada limite planetária com o modelo WRF-ARW
}

Vertical wind profile simulations in the planetary boundary layer with the WRF-ARW model

\author{
E. A. Lucas ${ }^{1 *}$; A. G. Arce' ; M. R. de Moraes²; G. C. Boezio ${ }^{1}$ \\ ${ }^{1}$ Facultad de Ingeniería (FING)/Instituto de Mecánica de los Fluidos e Ingeniería Ambiental (IMFIA)/Universidad de \\ la República (UdelaR), Montevideo, Uruguay \\ ${ }^{2}$ Laboratório de Modelagem e Simulaçao Computacional (LMSC), Universidade Federal do Pampa \\ (Unipampa),CEP:96461-172, Bagé/Rio Grande do Sul, Brasil \\ *ealmeida@fing.edu.uy
}

(Recebido em 28 de abril de 2017; aceito em 22 de maio de 2017)

\begin{abstract}
O presente trabalho analisa o comportamento do perfil vertical do vento nos primeiros 100 metros da camada limite planetária, através da análise de dados observados em uma torre de medição meteorológica no Uruguai, assim como dados simulados pelo modelo numérico WRF-ARW, os quais compreendem o período de 1 ano. Da torre de medição analisada (Colonia Eulacio), foram utilizadas informações referentes a velocidade média do vento em intervalos horários para as alturas de 10.1 metros e 101.8 metros. O modelo WRF-ARW foi executado para o mesmo período dos dados analisados, e configurado com distintas combinações de parametrizações físicas de camada limite planetária e camada limite superficial, buscando assim identificar dentre as diferentes simulações realizadas, qual o comportamento das mesmas em estimar a velocidade média do vento nos períodos anual, estacional durante o ciclo diário para as duas distintas alturas de interesse. Todas as parametrizações analisadas apresentaram um bom ajuste ao ciclo diário quando se comparou o nível eta correspondente a altura de 101.8 metros. Quando se analisou o ciclo diário do nível eta correspondente a 10.1 metros, somente a parametrização física de camada limite planetária Mellor-Yamada-Janjic conseguiu representar os aumentos de velocidade média do vento durante períodos diurnos em todas as estações do ano (inverno, primavera, verão e outono), resultando em uma maior correlação linear e menor erro média absoluto da mesma em relação as outras parametrizações físicas utilizadas no modelo.

Palavras chave: Perfil vertical do vento, camada limite planetária, ciclo diário, modelo WRF-ARW.
\end{abstract}

The aim of this article is to analyze the behaviour of the wind speed vertical profile for the first 100 meters of the planetary boundary layer. This is done by analyzing 1 year of ARW-WRF (Weather Research and Forecasting) model outputs as well as anemometric measurements from a tower installed in Uruguay. Colonia Eulacio tower, the tower used, measures wind speed at heights of 10.1 meters and 101.8 meters. In this article, mean hourly wind speeds at each height are used. Different parametrizations of surface layer and planetary boundary layers were set to ARW-WRF modelations, in order to compare their capability to model hourly mean wind speeds from tower measurements considering $101.8 \mathrm{~m}$ as well as $10.1 \mathrm{~m}$ height data. Such comparison was done for the whole year as well as for each season. A day-cicle comparison was also performed. All the parametrizations considered showed a good modelation of the Day-cicle for 101.8 meters height data. Regarding 10.1 meters height data, only Mellor-Yamada-Janjic (MYJ) showed a proper modelation of the day-cicle, being the only parametrization capable of representing correctly the mean speed rise during the day for all seasons. As a consequence, MYJ showed the biggest linear correlation factor as well as the lower mean absolute error.

Keywords: Wind speed vertical profile, Planetary boundary layer, ARW-WRF model. 


\section{INTRODUÇÃO}

Atualmente o estudo sobre o comportamento do vento dentro da camada limite planetária tem sido objeto de pesquisa de grande interesse para a área de energia, relacionado principalmente ao estudo da energia eólica. Neste contexto a utilização de modelos numéricos de circulação geral da atmosfera, como o WRF-ARW [1], tem ajudado a planejar e administrar questões relacionadas a exploração da energia eólica. Dentre as utilizações destes modelos, destacam-se os estudos de viabilidade de implantação de parques ou complexos eólicos, e a previsão de geração de energia elétrica desses empreendimentos.

Na previsão de geração de energia, destaca-se o desenvolvimento de ferramentas de tomada de decisões para empresas elétricas, as quais necessitam de informações prévias da produção total de energia dos parques eólicos para realizar de maneira racional o despacho de energia elétrica, diminuindo assim os custos de geração de energia elétrica, visto que a energia eólica é altamente competitiva do ponto de vista econômico e ambiental. Dentre os países que a expansão de energia eólica no sistema elétrico tem sido relevante destaca-se o Uruguai, o qual se encontra em uma etapa crescente de incorporação deste tipo de energia em sua matriz energética. Está previsto que a potência eólica instalada ao final ano de 2016 representará aproximadamente $25 \%$ da potência total instalada no país. Mediante um acordo entre a empresa elétrica nacional, a "Administración Nacional de Usinas y Transmisiones Eléctricas" (UTE) e a universidade pública do país, a "Universidad de la Republica" (UdelaR), há se desenvolvido em âmbito acadêmico uma ferramenta de previsões operacionais para os parques eólicos. Esta ferramenta é repassada para a UTE, que faz uso da mesma para auxiliar no despacho de energia elétrica na rede de transmissão. A partir do ano de 2007, foram realizadas instalações de estações de medições no território uruguaio pela empresa elétrica UTE, de modo a prospectar a produção de energia eólica no país. Atualmente o Uruguai possui uma vasta rede de medições, compreendendo anemômetros, wind vanes, piranômetros e termômetros, os quais se encontram distribuídos por todas as regiões do país. Uma das características desta rede de medições disponível, é que a mesma possui medidas em distintas alturas da camada limite atmosférica, permitindo assim analisar o comportamento de perfil vertical do vento.

A análise do ciclo diário do vento é de muita importância para o entendimento do comportamento do mesmo na camada limite atmosférica ao longo do ano. Uma das características do comportamento do perfil do vento no ciclo diário está diretamente relacionado com a interação da radiação solar incidente na superfície terrestre, onde destaca-se a predominância de condições de atmosfera estável e instável durante a maior parte de um período diário. $\mathrm{Na}$ atmosfera estável, as quais se encontram normalmente durante a noite, a superfície possui uma temperatura menos elevada que a atmosfera, recebendo assim calor sensível da atmosfera. Em condições instáveis, normalmente encontrada durante o dia, a atmosfera próxima está recebendo calor da superfície terrestre, sendo que esta recebe o fluxo de radiação solar [2]. Condições de atmosfera instável caracterizam também processos de formação de turbulência de origem térmica, resultantes da troca de calor da superfície terrestre com a atmosfera, a qual interfere no perfil de velocidade do vento, onde se pode encontrar uniformidade com a altura no campo desta variável em condições de atmosfera instável [3].

Em um estudo realizado [4], observou que durante os momentos posteriores ao nascer do sol (instabilidade atmosférica) se observa uma dimuição na velocidade média nas camadas próximas a 100 metros de altura e um aumento na velocidade média do vento nas camadas com alturas menores que 25 metros. A intensidade de turbulência decresce com a altura para os diferentes estados de estabilidade associados ao ciclo diário, porém quando há uma comparação entre atmosferas estáveis e instáveis registra-se um maior valor de intensidade de turbulência nas condições de instabilidade atmosféricas.

Os modelos numéricos de circulação geral da atmosfera, como o WRF-ARW [1], buscam representar da melhor maneira através de parametrizações físicas todas os processos de interação entre superfície terrestre e atmosfera terrestre para as diferentes condições atmosféricas. Dentre as parametrizações físicas mais importantes que buscam descrever estes processos, destacam-se as de camada limite planetária e camada limite superficial, as quais são relacionadas conjuntamente em configurações de simulação/previsão no modelo numérico WRF-ARW. Dentre 
as parametrizações de camada limite atmosférica, a "Yonsei University" é um esquema de primeira ordem que utiliza alguns coeficientes de difusão turbulenta não locais para calcular os fluxos turbulentos.

Já algumas parametrizações de camada limite planetária como Mellor-Yamada-Janjic (MYJ), Bougeault-Lacarrère (Boulac) e Grenier-Bretherton-McCaa (GBM) buscam prever também a energia cinética turbulenta na camada limite planetária. Para as parametrizações de camada limite superficial, destacam-se "Revised MM5 Monin-Obukhov" e "MoninObukhov (Janjic Eta)". O esquema Revised MM5 Monin-Obukhov se baseia no esquema de Monin-Obukhov (Teoria da Similaridade de Monin-Obukhov). A descrição do escoamento médio e dos fluxos turbulentos em uma camada superficial horizontalmente homogênea depende somente de 4 variáveis independentes: altura $\mathrm{z}$, arrasto superficial, fluxo cinemático de calor na superfície e o parâmetro de empuxo. A parametrização Monin-Obukhov (Janjic Eta), também se baseia na teoria de similaridade de Monin-Obukhov e inclui parametrizações para uma subcamada viscosa sobre a água, assim como sobre o solo. Para o caso do solo, esta opção considera a altura da rugosidade variável com a temperatura e umidade. A mesma utiliza um método de iteração de cálculo para os fluxos de superfície na camada. Além disso, este esquema incorpora correções para evitar as singularidades no caso de uma camada superficial instável e uma velocidade de vento decrescente.

\section{MATERIAL E MÉTODOS}

\section{1 ÁREA DE ESTUDO}

A estação de medição utilizada para estudo está localizada na região sudoeste do Uruguai, no departamento de Soriano, mais especificamente na localidade de Colonia Eulacio. A mesma é composta por uma torre de seção triangular, com 100.8 metros de altura, 0.45 metros de largura e está localizada conforme Datum WGS84 em 33 16'S, 57 31' O. A altitude onde a mesma se encontra instalada é de aproximadamente 100 metros, e seu entorno é de campos com planícies, portanto caracterizando a mesma em lugar de terreno não complexo. A referida estação é de propriedade da "Administración Nacional de Usinas y Transmissiones Eléctricas" (UTE), sendo esta a empresa estatal Uruguaia responsável pela geração, distribuição e comercialização da energia elétrica no país. Para o presente estudo, foram utilizadas as medições de dois anemômetros (Marca NRG, modelo 40C do tipo "concha") instalados em duas distintas alturas de medições: 10.1 e 101.8 metros. O período de análise foi do dia 08 de Agosto de 2014 as 00:00 horas até 07 de Agosto de 2015 as 23:00 horas, totalizando 1 ano de dados de velocidade média do vento.

\subsection{PRÉ-PROCESSAMENTO DOS DADOS OBSERVADOS}

Buscando verificar a qualidade dos dados observados pelos dois anemômetros de interesse na torre Colonia Eulacio, foi realizado um pré-processamento dos mesmos, de modo a garantir a qualidade das medidas. Os dados obtidos pelo datalogger possuem medidas a cada 2 segundos, onde são armazenados os valores de leitura e feita uma média estatística a cada 10 minutos, de onde são apresentados o desvio padrão, valores máximos e valores mínimos das variáveis medidas. Os valores de interesse foram as médias da velocidade do vento registradas a cada 10 minutos. Na torre Colonia Eulacio, os anemômetros localizados a 101.8 metros são em número de dois, com diferença de disposição de 180 graus do anemômetro "I A" para o anemômetro "I B". Já para a altura de 10.1 metros há apenas um anemômetro para realizar as medições. Esta configuração na disposição dos anemômetros na torre de medição foi realizada, buscando evitar os efeitos esteira causado pela torre de medição, quando da passagem do vento pela mesma, sendo para isto considerada a norma IEC 61400-12-1 (2005), intitulada como "Power performance measurements of electricity producing wind turbines" trata em seu anexo G "Mounting of instruments on the meteorological mast" as condições a serem consideradas para a instalação de instrumentos de medições em uma torre, sejam estas de seção quadrada, triangular ou circular. Para 10.1 metros de altura, a distância do anemômetro em relação a estrutura da torre foi essencial 
para que o mesmo não obtivesse medidas errôneas causadas por efeito esteira da torre, sendo analisados os dados da mesma. A orientação dos anemômetros localizados nas alturas de $10.1 \mathrm{e}$ 101. metros é mostrado na Tabela 1 abaixo.

Tabela 1: Orientação (graus) dos anemômetros localizados a 10.1 e 101. metros de altura.

\begin{tabular}{|c|c|c|}
\hline Instrumento & Altura (metros) & Orientação Geográfica (graus) \\
\hline Anemômetro IA & 101.8 & 350 \\
\hline Anemômetro IIA & 101.8 & 170 \\
\hline Anemômetro II & 10.1 & 24 \\
\hline
\end{tabular}

Sendo assim as medições para a altura dos anemômetros a 101.8 metros foram validadas por um anemômetro "fíctício", o qual considerava durante determinadas direções do vento as medidas do instrumento I A, e em determinadas direções considerava apenas as medidas do I B, mantendo assim as medidas sem influências da estrutura da torre. A configuração da montagem e da torre sobre a que se encontram os instrumentos de medição influenciam sensivelmente na qualidade e confiabilidade dos dados obtidos pelos mesmos. Após identificadas as medições que não foram afetadas pelo efeito esteira da torre, foram verificadas a presença ou não de dados faltantes, e logo após verificado as suas magnitudes, buscando identificar possíveis problemas de leitura dos anemômetros, como "travamentos" por exemplo. Por fim os dados de 1 ano observados em intervalo de 10 em 10 minutos, foram convertidos para valores horários, sendo realizado as médias das medidas originais medidas no intervalo de 60 minutos (1 hora), obtendo desta maneira medições no mesmo intervalo de tempo de saída do modelo WRF-ARW, os quais foram comparados posteriormente.

\subsection{SIMULAÇÕES WRF-ARW}

O modelo numérico de circulação atmosférica WRF-ARW versão 3.7.1 foi utilizado para simular o perfil vertical do vento para o mesmo período dos dados observados na estação de medição Colonia Eulacio. O modelo foi configurado com 2 domínios de resolução horizontal de $18 \mathrm{~km}$ e $6 \mathrm{~km}$ respectivamente, com números de pontos de malha contendo 101x101 (d01) pontos e 106x112 (d02), sendo a projeção cartográfica utilizada Lambert Conformal. A Figura 1 abaixo, mostra a disposição dos domínios utilizados nas simulações realizadas, assim como a localização da torre Colonia Eulacio.
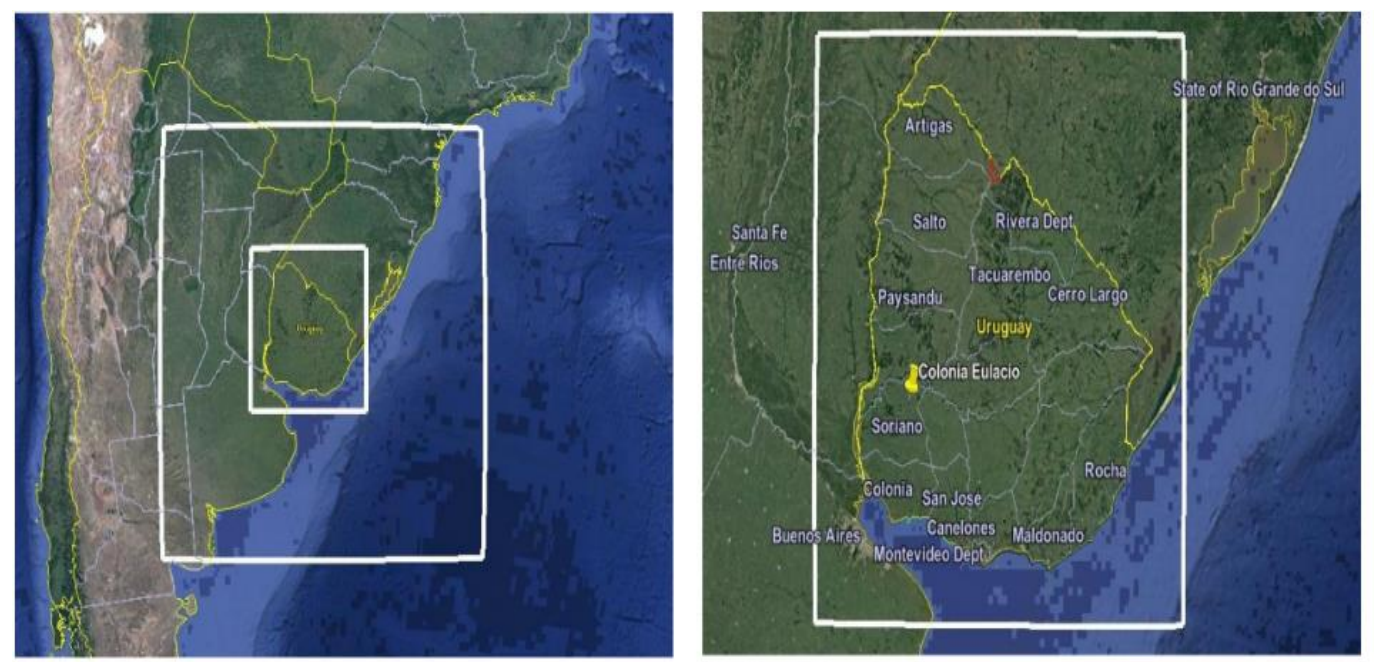

Figura 1: Domínios utilizados para as simulações com uso do modelo WRF-ARW (à esquerda) $e$ localização da estação de medição Colonia Eulacio (à direita).

Os dados de condição de contorno utilizados foram do Global Forecasting System (GFS) de resolução 0.5 (aproximadamente $56 \mathrm{~km}$ ), o modelo de uso do solo foi o "MODIS 2010" da 
Fing/UdelaR, que possui 20 classes de uso do solo e resolução espacial de 500 metros. Os níveis verticais do modelo (eta levels) foram configurados em número de 41, possuindo uma distribuição de alta resolução nos primeiros 150 metros da camada limite planetária.

Quanto as parametrizações físicas do modelo, algumas foram "fixas" para todas as simulações realizadas, as quais destacam-se: Lin Purdue (microfísica), RRTM (radiação de onda longa), Dudhia (radiação de onda curta) e Kain Fritsh (cumulus). Para as parametrizações de camada limite planetária e camada limite superficial, foram utilizadas algumas combinações, totalizando 5 distintas configurações, as quais foram simuladas para o mesmo período de interesse, sendo as mesmas apresentadas na Tabela 2.

Tabela 2: Distintas configurações de parametrizações de camada limite planetária $(C L P)$ e camada limite superficial (CLS) utilizadas nas simulações com uso do modelo WRF-ARW.

\begin{tabular}{|c|c|}
\hline Parametrização de CLP & Parametrização de CLS \\
\hline Yonsei University (Yonsei) & Revised MM5 \\
\hline Mellor-Yamada-Janjic (MYJ) & Janjic Eta \\
\hline Bougeault-Lacarrère (Boulac) & Revised MM5 \\
\hline Bougeault-Lacarrère (Boulac) & Revised MM5 \\
\hline Grenier-Bretherton-McCaa (GBM) & Revised MM5 \\
\hline
\end{tabular}

\section{RESULTADOS E DISCUSSÃO}

\subsection{DADOS OBSERVADOS}

Analisando-se os dados horários observados na estação Colonia Eulacio para as alturas de 10.1 metros e 101.8 metros obteve-se uma velocidade média do vento anual de $4.02 \mathrm{~m} / \mathrm{s}$ para altura de 10.1 metros e $7.21 \mathrm{~m} / \mathrm{s}$ para a altura de 101.8 metros. Em períodos estacionais as velocidades médias são apresentadas na Figura 2.

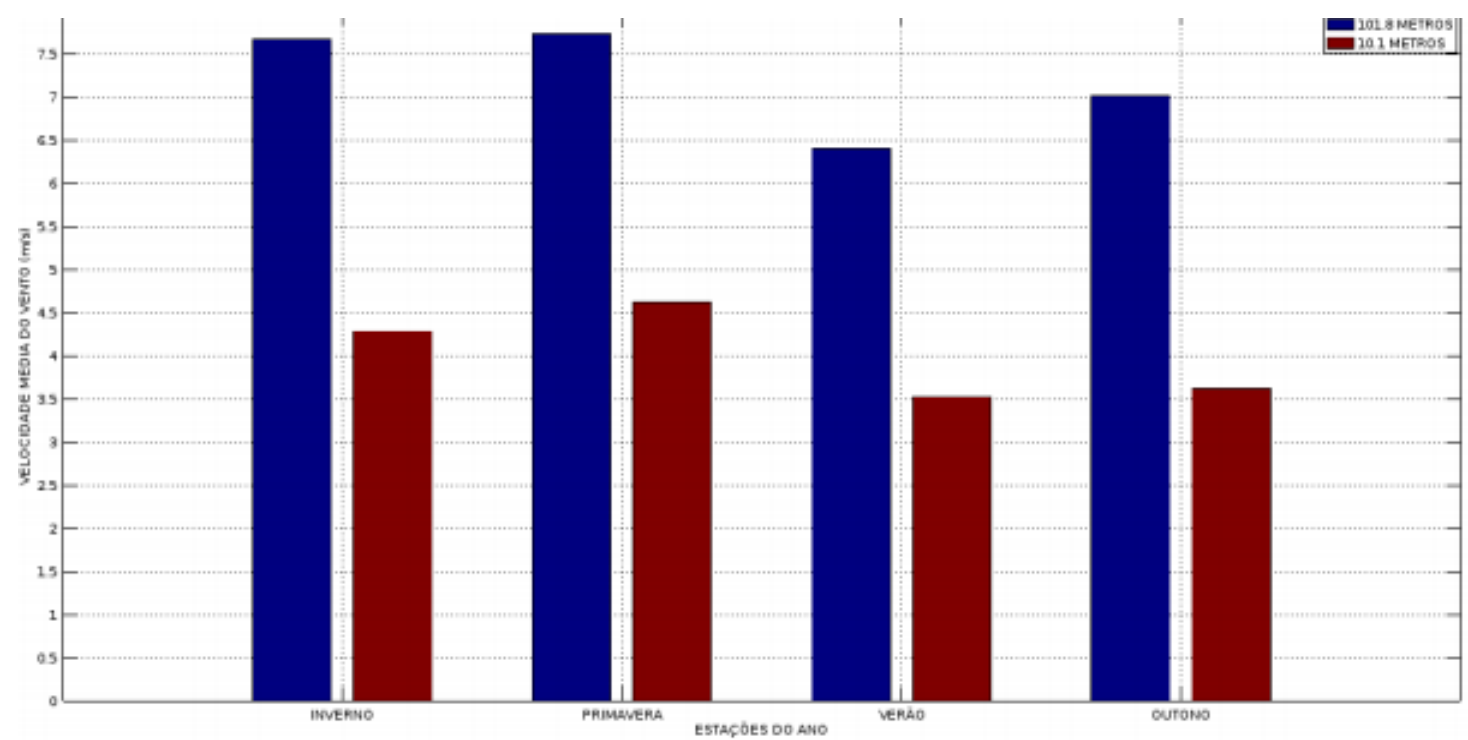

Figura 2: Velocidades médias do vento estacionais para as alturas de 10.1 metros e 101.8 metros de altura na torre Colonia Eulacio.

De acordo com a Figura 2, as maiores velocidades médias do vento na estação Colonia Eulacio, foram identificadas nas estações do inverno e primavera, e as menores para as estações do verão e outono. 


\subsection{DADOS SIMULADOS PELO MODELO WRF-ARW}

Para analisar os resultados simulados pelo modelo WRF-ARW, foi realizada uma análise estatística baseada no erro médio absoluto (EMA) da velocidade média do vento (m/s), assim como a correlação linear entre os resultados de saída do modelo para o domínio de maior resolução espacial $(6 \mathrm{~km})$ com os dados observados na torre Colonia Eulacio. As análises realizadas para as alturas de 10.1 metros e 101.8 metros, basearam-se em saídas do modelo para as componentes do vento, nos níveis verticais do modelo correspondente as alturas de interesse.

\subsection{ANÁlISE PARA A ALTURA DE 10.1 METROS}

A Tabela 3 mostra as distintas simulações realizadas, e seus respectivos valores de erro médio absoluto (EMA) e correlação linear correspondentes a altura de 10.1 metros para todo o período analisado (1 ano).

Tabela 3: Distintas configurações de parametrizações de camada limite planetária (CLP) e camada limite superficial (CLS) utilizadas nas simulações com uso do modelo WRF-ARW.

\begin{tabular}{|c|c|c|c|}
\hline Parametrização de CLP & Parametrização de CLS & EMA(m/s) & Correlação \\
\hline $\begin{array}{c}\text { Yonsei University } \\
\text { (Yonsei) }\end{array}$ & Revised MM5 & 1.09 & 0.72 \\
\hline $\begin{array}{c}\text { Mellor-Yamada-Janjic } \\
\text { (MYJ) }\end{array}$ & Janjic Eta & 1.04 & 0.76 \\
\hline $\begin{array}{c}\text { Bougeault-Lacarrère } \\
\text { (Boulac) }\end{array}$ & Revised MM5 & 1.26 & 0.69 \\
\hline $\begin{array}{c}\text { Bougeault-Lacarrère } \\
\text { (Boulac) }\end{array}$ & Revised MM5 & 1.14 & 0.73 \\
\hline $\begin{array}{c}\text { Grenier-Bretherton- } \\
\text { McCaa (GBM) }\end{array}$ & Revised MM5 & 1.12 & \\
\hline
\end{tabular}

A Figura 3 mostra o comparativo entre os dados observados e os dados simulados com as cinco distintas configurações de parametrizações físicas, para período de 1 ano, focando na análise do ciclo diário da velocidade do vento para a altura de 10.1 metros.
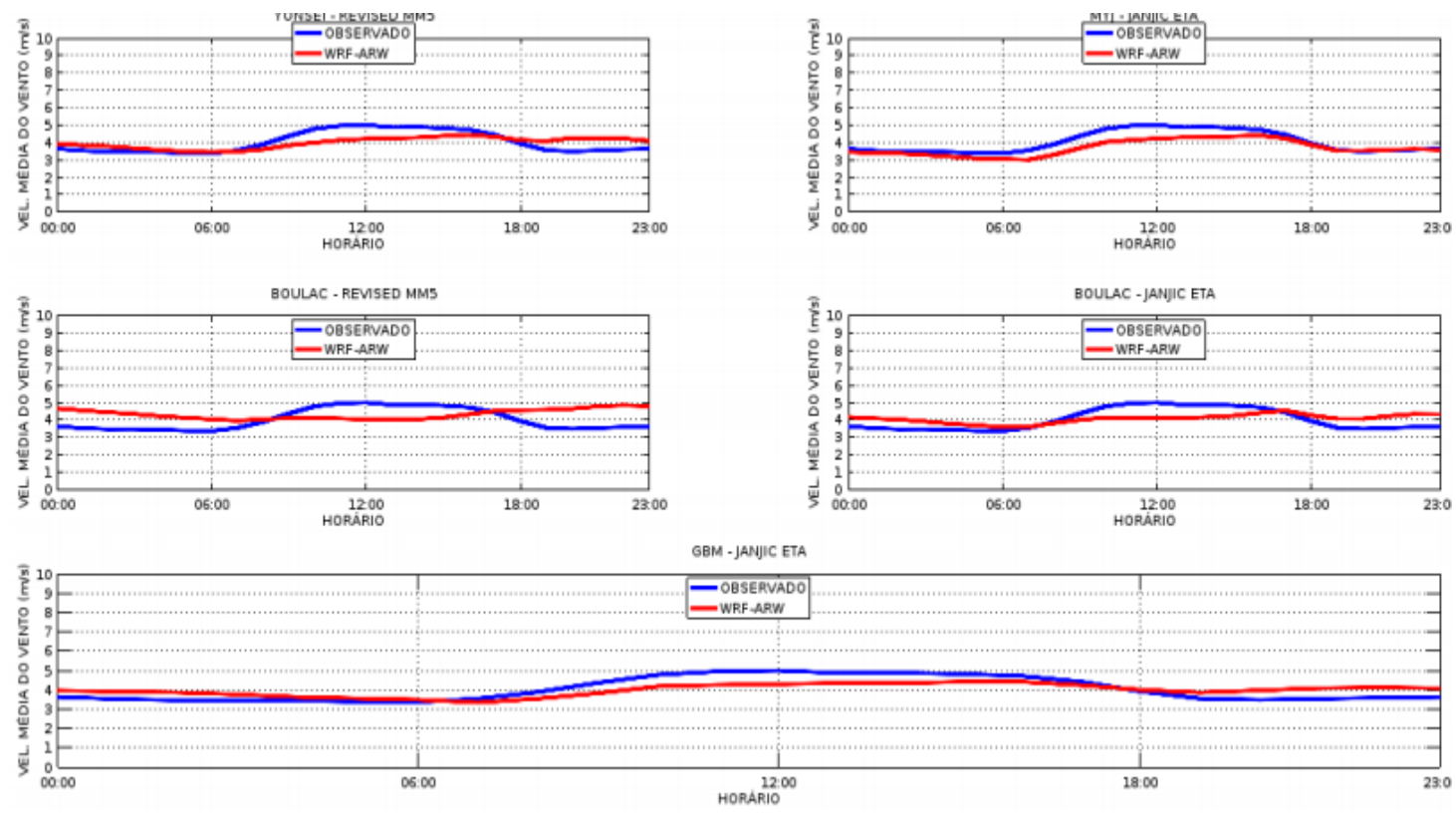

Figura 3: Velocidades médias do vento anual para a altura de 10.1 metros.

Conforme ilustrado na figura 3, percebe-se que para todas as cinco simulações realizadas as velocidades médias do vento durante o período diurno foram subestimadas em relação a média 
anual observada na torre Colonia Eulacio para o mesmo período. Para o período noturno a combinação de parametrizações Mellor-Yamada-Janjic (camada limite planetária) e Janjic Eta (camada limite superficial) obteve uma estimativa de velocidade média do vento bem próxima da observada para o mesmo período. As outras quatro combinações de parametrizações utilizadas, obtiveram de forma geral uma tendência de sobreestimar as velocidades médias do vento para o período noturno. Sabendo do comportamento distinto da velocidade média do vento ao longo das quatro estações do ano, foi analisado o comportamento das simulações para o inverno, primavera, verão e outono. O comparativo entre os dados simulados e dados observados para a altura de 10.1 metros na estação do inverno é mostrado na Figura 4 abaixo.
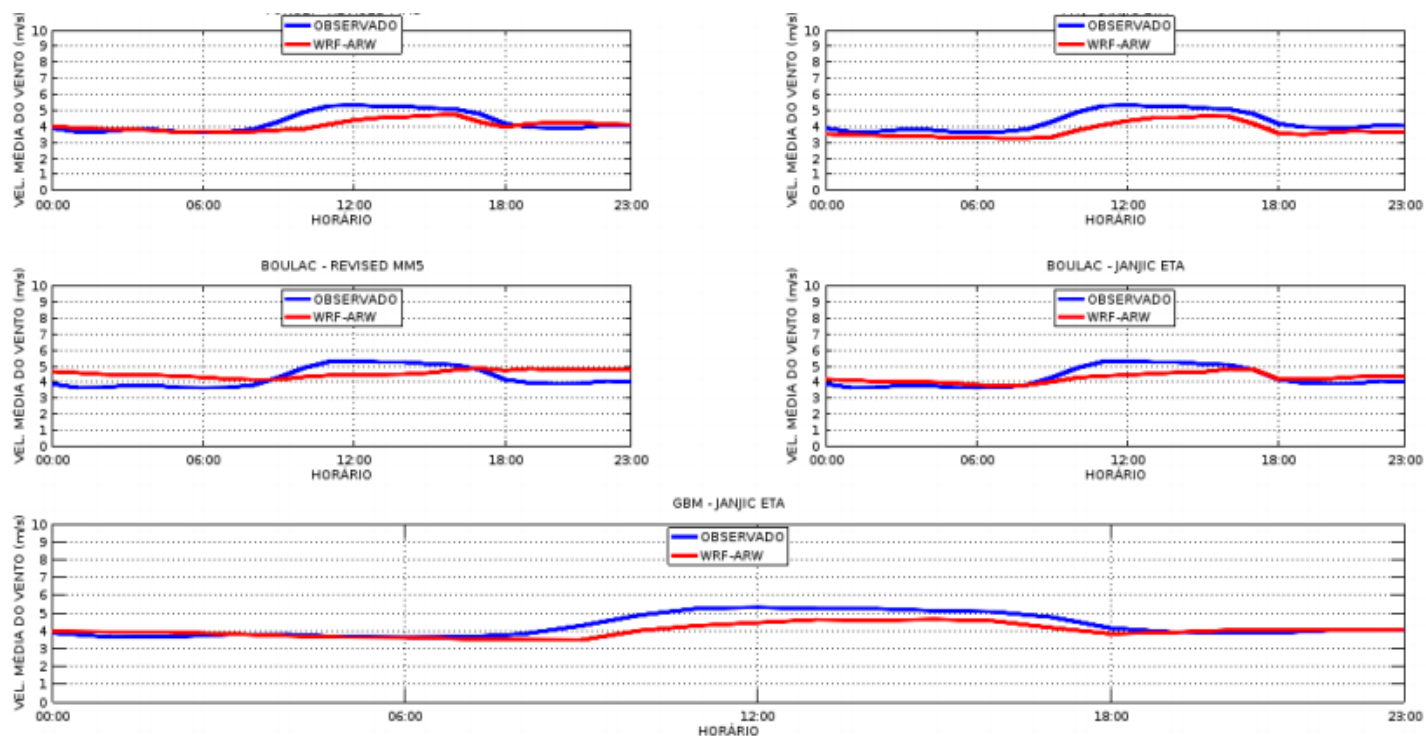

Figura 4: Velocidades médias do vento no inverno observadas na torre Colonia Eulacio (linha azul) e simuladas pelas distintas parametrizações utilizadas no modelo WRF-ARW (linha vermelha) para a altura de 10.1 metros.

Conforme a Figura 4, percebe-se que todas as parametrizações subestimam na estação do inverno as velocidades médias do vento para o período diurno. Já para o período noturno apenas a combinação de parametrizações Boulac-Revised MM5 obtiveram valores de velocidade média que não foram próximos dos observados para o mesmo período. O comparativo entre os dados simulados e dados observados para a altura de 10.1 metros na estação da primavera é mostrado na Figura 5.
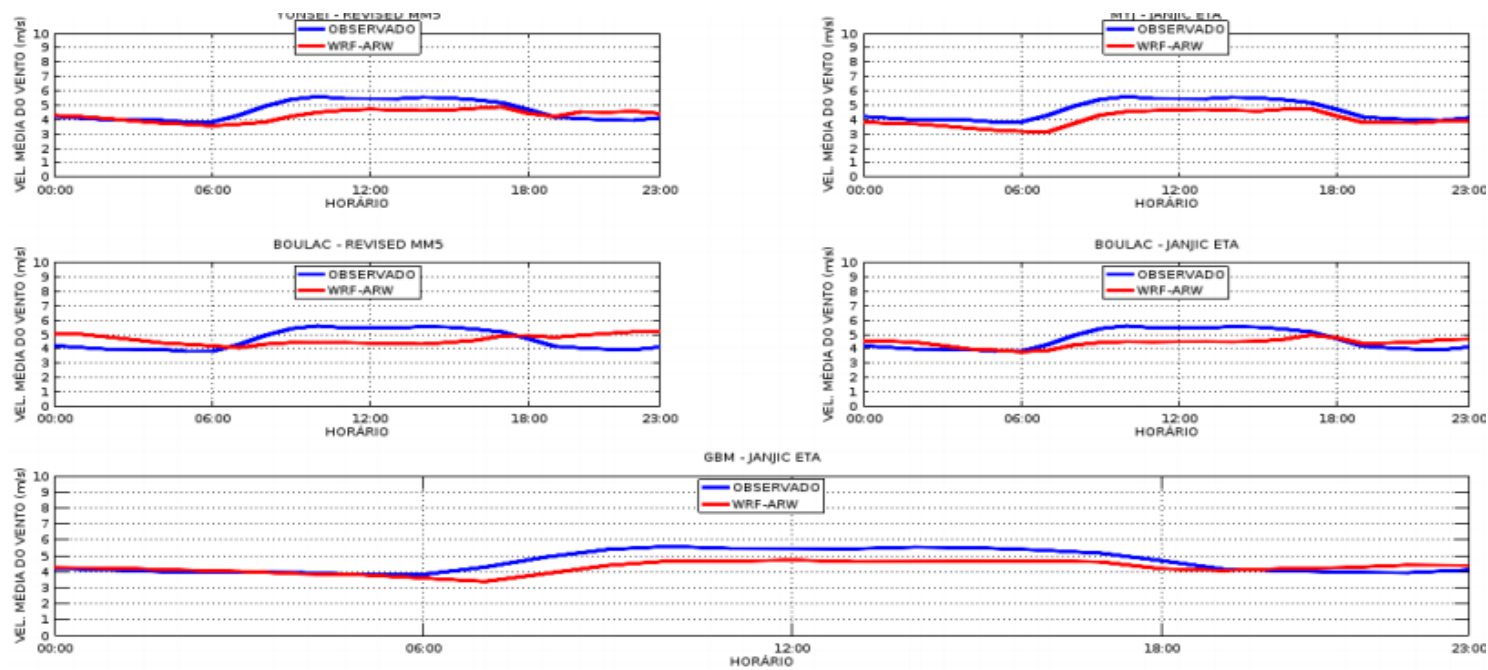

Figura 5: Velocidades médias do vento na primavera observadas na torre Colonia Eulacio (linha azul) e simuladas pelas distintas parametrizações utilizadas no modelo WRF-ARW (linha vermelha) para a altura de 10.1 metros. 
Conforme a Figura 5, percebe-se que todas as parametrizações subestimam na estação da primavera as velocidades médias do vento para o período diurno. Já para o período noturno, percebe-se que a combinação de parametrizações GBM - Janjic Eta estima de maneira satisfatória as magnitudes das velocidades médias. Para o mesmo período noturno da estação primavera, as quatro combinações de parametrizações restantes não obtiveram a mesma acurácia da combinação citada anteriormente. O comparativo entre os dados simulados e dados observados para a altura de 10.1 metros na estação verão é mostrado na Figura 6.
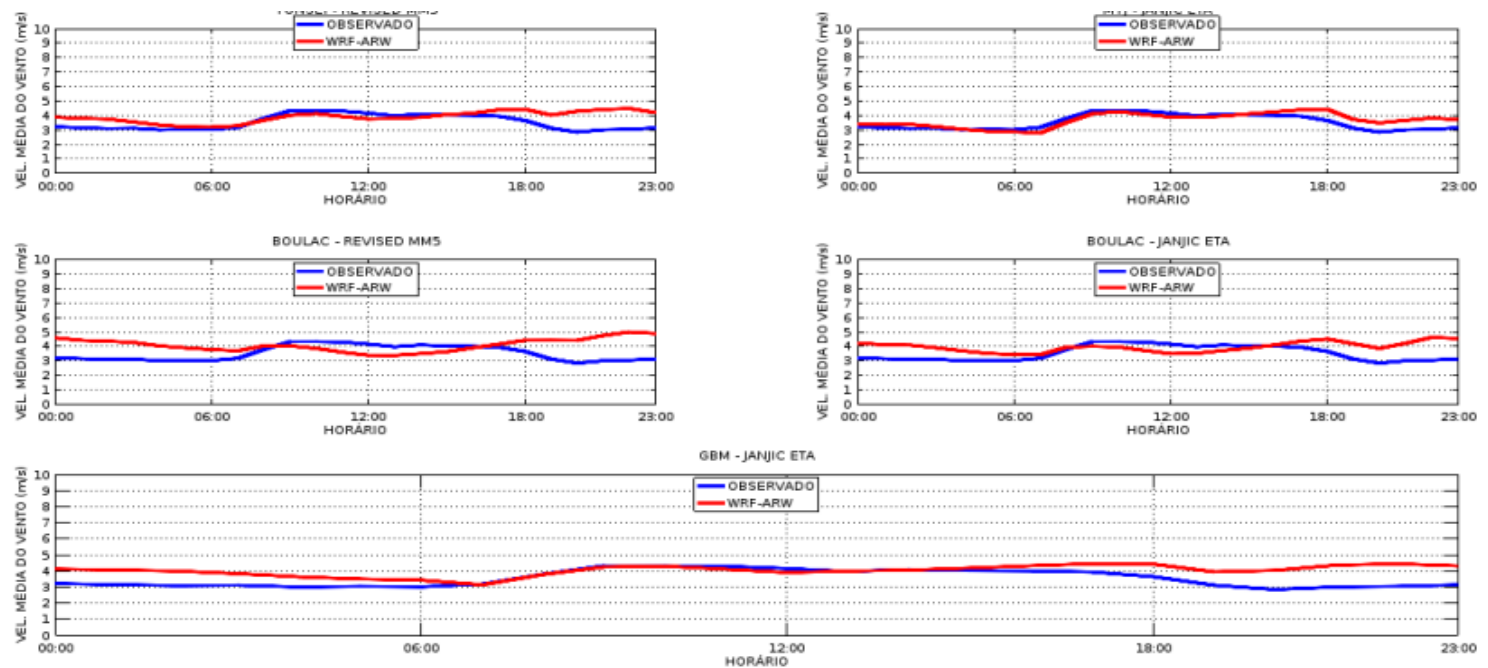

Figura 6: Velocidades médias do vento no verão observadas na torre Colonia Eulacio (linha azul) e simuladas pelas distintas parametrizações utilizadas no modelo WRF-ARW (linha vermelha) para a altura de 10.1 metros.

Conforme a Figura 6, percebe-se que para o período diurno, a combinação de parametrizações Yonsei-Revised MM5, MYJ-Janjic Eta e GBM-Janjic Eta obtiveram boa estimativa das velocidades médias do vento para a estação do verão. Para o período noturno, percebe-se que a combinação de parametrizações MYJ - Janjic Eta obteve uma melhor aproximação da velocidade média observada para a altura de 10.1 metros na estação do verão, sendo que as parametrizações restantes tiveram a tendência de superestimar os valores. Por fim, é mostrado o comparativo entre os dados simulados e dados observados para a altura de 10.1 metros na estação do outono, o qual é mostrado na Figura 7.
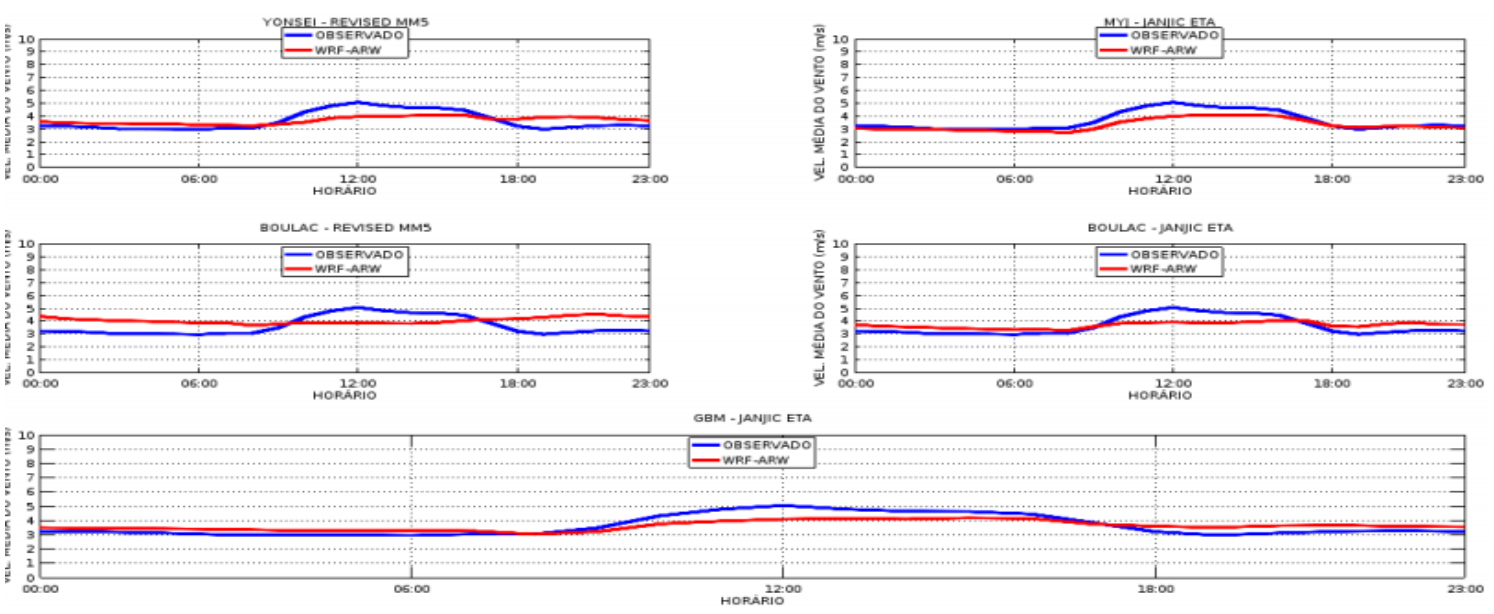

Figura 7: Velocidades médias do vento no outono observadas na torre Colonia Eulacio (linha azul) e simuladas pelas distintas parametrizações utilizadas no modelo WRF-ARW (linha vermelha) para a altura de 10.1 metros.

Conforme a Figura 7, percebe-se que para o período diurno, todas as combinações de parametrizações subestimaram os valores observados para a altura de 10.1 metros na estação do 
outono. Para o período noturno, percebe-se que a combinação de parametrizações MYJ-Janjic Eta obteve uma melhor aproximação da velocidade média observada.

\subsection{ANÁLISE PARA A ALTURA DE 101.8 METROS}

Uma análise detalhada para a altura de 101.8 metros é realizada, pois além de ser possível verificar o comportamento do ciclo diário da velocidade do vento nesta altura e compará-la com a altura de 10.1 metros, é possível estabelecer uma idéia de como varia a produção de energia eólica ao longo do dia, em período anual e períodos estacionais, visto que todos os parques eólicos no Uruguai possuem alturas do rotor próximas a 100 metros.

A Tabela 4 mostra as distintas simulações realizadas, e seus respectivos valores de erro médio absoluto (EMA) e correlação linear correspondentes a altura de 101.8 metros para todo o período analisado (1 ano).

Tabela 4: Distintas configurações de parametrizações de camada limite planetária $(C L P) e$ camada limite superficial (CLS) utilizadas nas simulações com uso do modelo WRF-ARW.

\begin{tabular}{|c|c|c|c|}
\hline Parametrização de CLP & Parametrização de CLS & EMA(m/s) & Correlação \\
\hline $\begin{array}{c}\text { Yonsei University } \\
\text { (Yonsei) }\end{array}$ & Revised MM5 & 1.59 & 0.73 \\
\hline $\begin{array}{c}\text { Mellor-Yamada-Janjic } \\
\text { (MYJ) }\end{array}$ & Janjic Eta & 1.60 & 0.74 \\
\hline $\begin{array}{c}\text { Bougeault-Lacarrère } \\
\text { (Boulac) }\end{array}$ & Revised MM5 & 1.71 & 0.75 \\
\hline $\begin{array}{c}\text { Bougeault-Lacarrère } \\
\text { (Boulac) }\end{array}$ & Revised MM5 & 1.77 & 0.74 \\
\hline $\begin{array}{c}\text { Grenier-Bretherton- } \\
\text { McCaa (GBM) }\end{array}$ & Revised MM5 & 1.55 & \\
\hline
\end{tabular}

Conforme apresentado na Tabela 4, percebe-se que os valores de erro médio absoluto são maiores em magnitude quando comparados aos mesmos para a altura de 10.1 metros. As configurações de simulação que utilizaram a parametrização de camada limite planetária Boulac, obtiverem valores de erro médio absoluto consideravelmente maiores do que as outras três configurações restantes. As correlações lineares entre as cinco distintas configurações utilizadas possuem valores muito próximos quando analisadas para o período anual. Para a altura de 101.8 metros no período anual, percebeu-se que para todas as cinco simulações realizadas as velocidades médias do vento durante o período diurno foram subestimadas em relação a média anual observada na torre Colonia Eulacio para o mesmo período. Para o período noturno as combinações de parametrizações Yonsei-Revised MM5, MYJ-Janjic Eta, GBM-Janjic Eta obtiveram uma estimativa de velocidade média do vento bem próxima da observada. Para as combinações de parametrizações de camada limite planetária e camada limite superficial BoulacRevised MM5 e Boulac-Janjic Eta, o modelo WRF-ARW subestimou os valores médios de velocidade do vento para o período noturno, seguindo a mesma tendência encontrada para o período diurno. A Figura 8 mostra o comparativo entre os dados observados e os dados simulados com as cinco distintas configurações, para período de 1 ano, focando na análise do ciclo diário da velocidade do vento para a altura de 101.8 metros. 

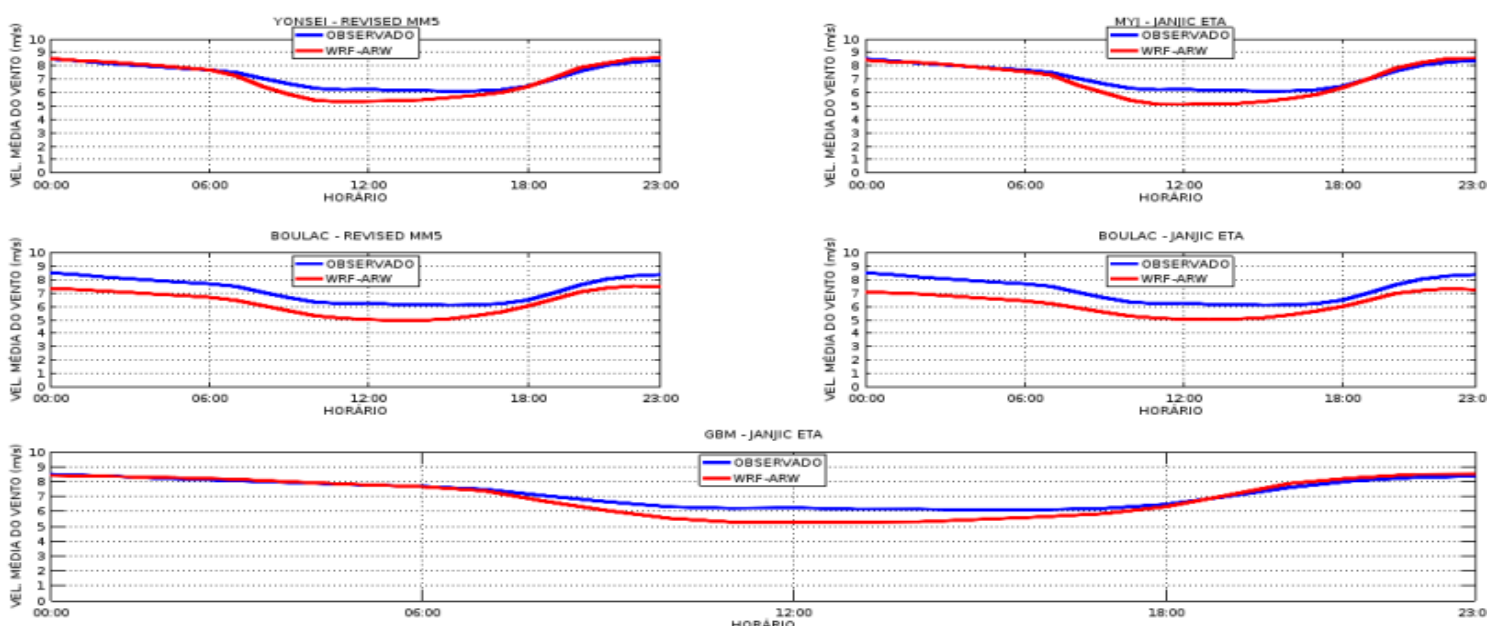

Figura 8: Velocidades médias do vento anual observadas na torre Colonia Eulacio (linha azul) e simuladas pelas distintas parametrizações utilizadas no modelo WRF-ARW (linha vermelha) para a altura de 101.8 metros.

O comparativo entre os dados simulados pelo modelo WRF-ARW e dados observados para a altura de 101.8 metros na estação do inverno é mostrado na Figura 9.
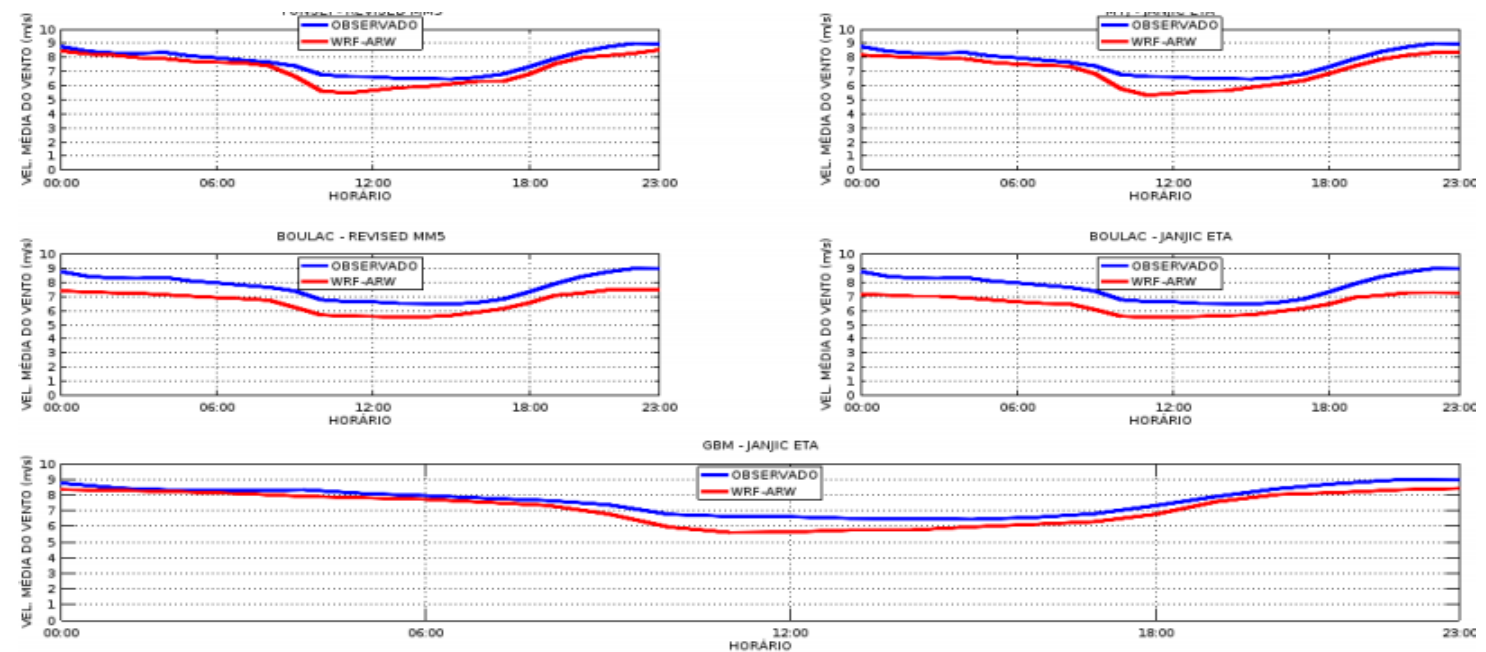

Figura 9: Velocidades médias do vento no inverno observadas na torre Colonia Eulacio (linha azul) e simuladas pelas distintas parametrizações utilizadas no modelo WRF-ARW (linha vermelha) para a altura de 101.8 metros.

Conforme a Figura 9, percebe-se que todas as parametrizações subestimam na estação do inverno as velocidades médias do vento para o período diurno. Já para o período noturno é possível observar que a combinação de parametrizações GBM - Janjic Eta obteve valores bem próximos aos observados, enquanto que as parametrizações Yonsei-Revised MM5 e MYJ-Janjic Eta subestimaram levemente as médias noturnas para o inverno. Para as combinações de parametrizações de camada limite planetária e camada limite superficial Boulac-Revised MM5 e Boulac-Janjic Eta, o modelo WRF-ARW subestimou consideravelmente os valore médios de velocidade do vento no inverno, para o período noturno. Para a estação da primavera, analisandose os resultados foi possível perceber que todas as parametrizações subestiram nas velocidades médias do vento para o período diurno. Para o período noturno, três combinações de parametrizações obtiveram estimativas bem próximas aos valores observados, as quais foram Yonsei-Revised MM5, MYJ-Janjic Eta e GBM - Janjic Eta. Para as combinações de parametrizações de camada limite planetária e camada limite superficial Boulac-Revised MM5 e Boulac-Janjic Eta, o modelo WRF-ARW subestimou consideravelmente os valore médios de velocidade do vento na primavera, para o período noturno. O comparativo entre os dados 
simulados e dados observados para a altura de 101.8 metros na estação da primavera é mostrado na Figura 10.
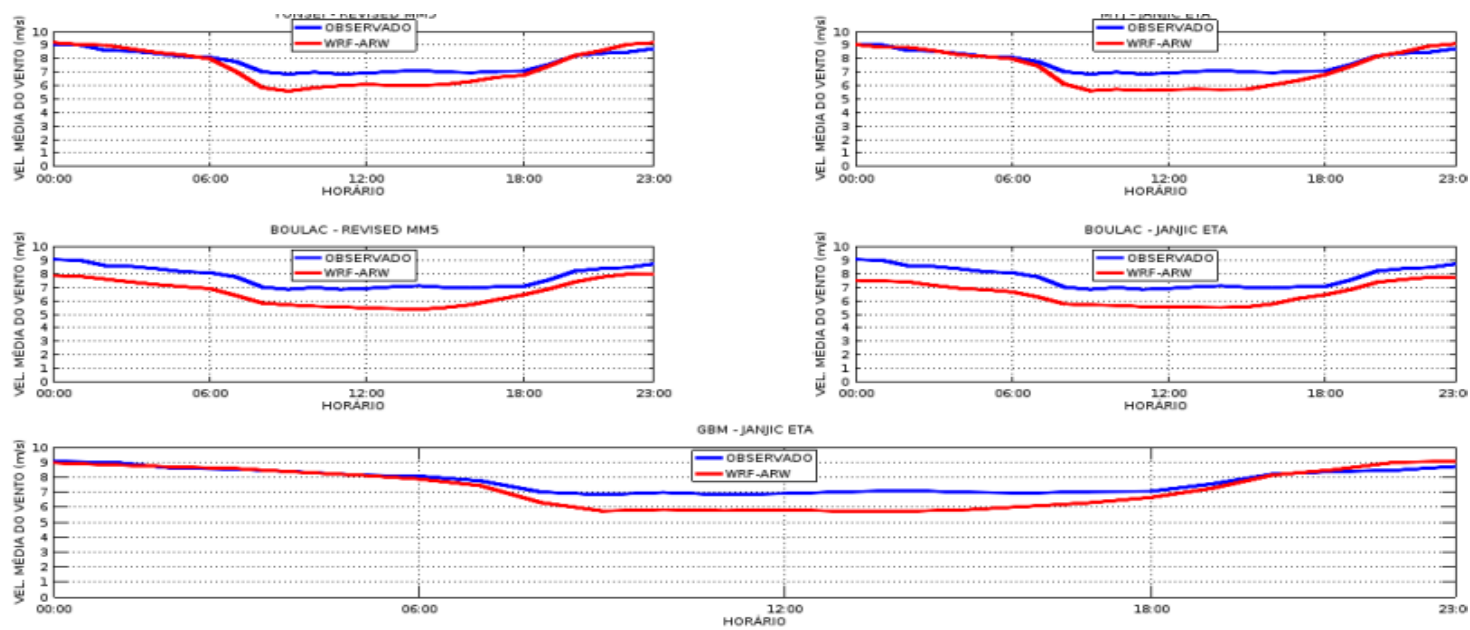

Figura 10: Velocidades médias do vento na primavera observadas na torre Colonia Eulacio (linha azul) e simuladas pelas distintas parametrizações utilizadas no modelo WRF-ARW (linha vermelha) para a altura de 101.8 metros.

Para a estação do verão, analisando-se os resultados para o período diurno, foi possível perceber que a configuração que utilizou as parametrizações físicas de camada limite planetária a camada limite superficial Yonsei-Revised MM5 obteve uma leve subestimação dos valores observados, porém com aproximações satisfatórias. Quanto as outras combinações utilizadas, todas subestimaram significativamente os resultados quando comparadas com Yonsei-Revised MM5. Para o período noturno, três combinações de parametrizações sobreestimaram os valores observados, as quais foram Yonsei-Revised MM5, MYJ-Janjic Eta e GBM - Janjic Eta. Para as combinações de parametrizações de camada limite planetária e camada limite superficial BoulacRevised MM5 e Boulac-Janjic Eta, o modelo WRF-ARW subsestimou os valores médios de velocidade do vento no verão, para o período noturno. O comparativo entre os dados simulados e dados observados para a altura de 101.8 metros na estação verão é mostrado na Figura 11.
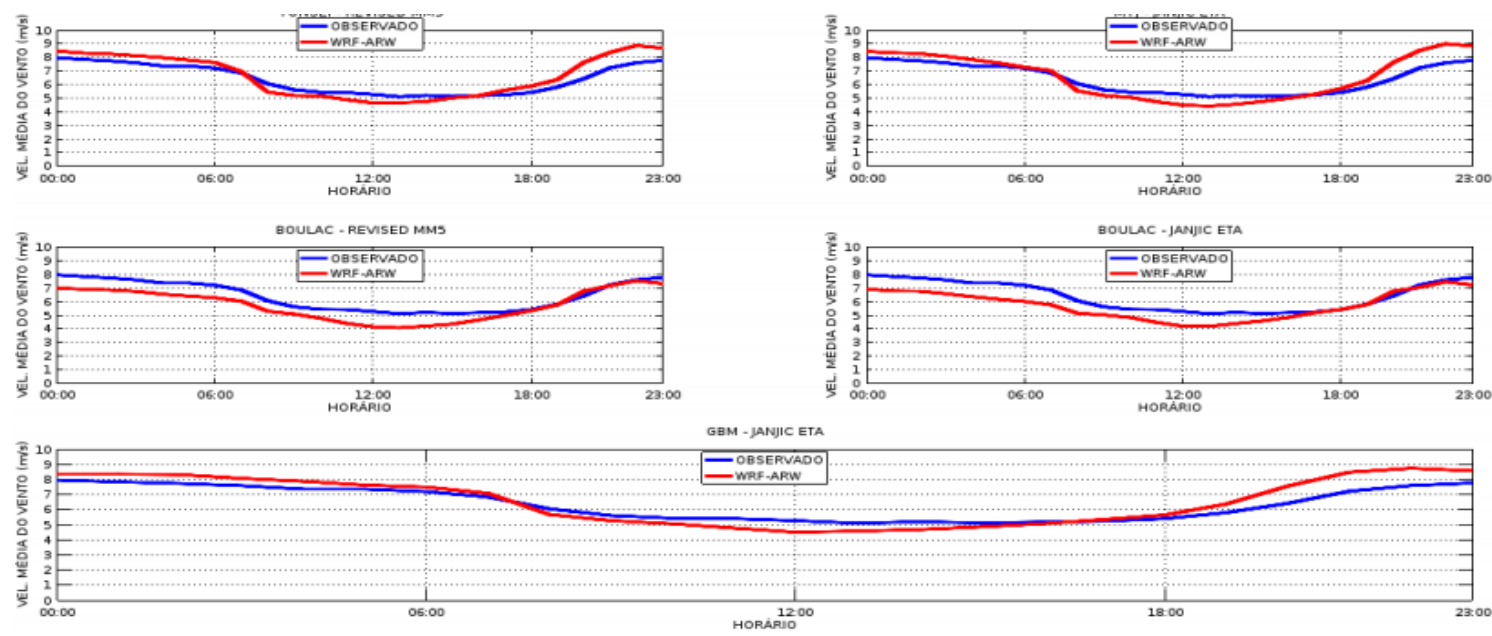

Figura 11: Velocidades médias do vento no verão observadas na torre Colonia Eulacio (linha azul) e simuladas pelas distintas parametrizações utilizadas no modelo WRF-ARW (linha vermelha) para a altura de 101.8 metros

Para a estação do outono, analisando-se o período diurno foi possível perceber que todas as combinações de parametrizações físicas utilizadas subestimaram em velocidade média do vento os valores observados. Para o período noturno, três combinações de parametrizações obtiveram bons resultados (Yonsei-Revised MM5, MYJ-Janjic Eta e GBM-Janjic Eta). Para as combinações 
de parametrizações Boulac-Revised MM5 e Boulac-Janjic Eta, o modelo WRF-ARW subestimou os valores médios de velocidade do vento no outono, para o período noturno. Na Figura 12 é mostrado o comparativo entre os dados simulados e dados observados para a altura de 101.8 metros na estação do outono.
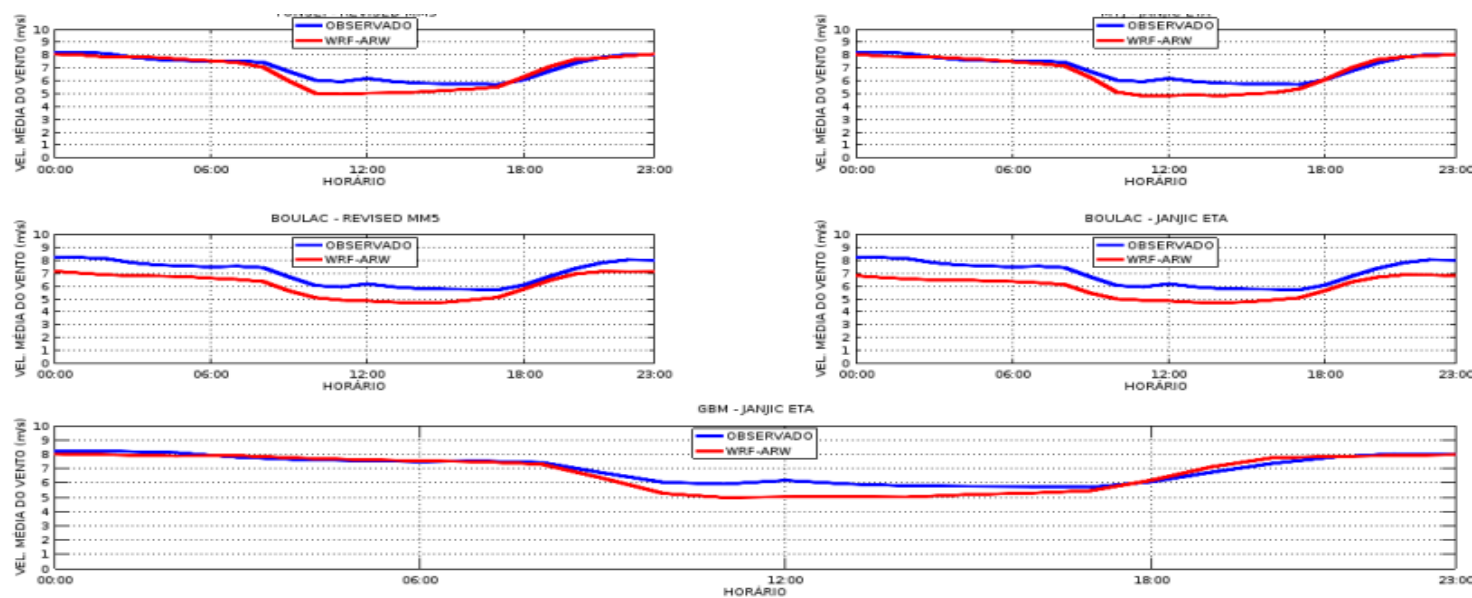

Figura 12: Velocidades médias do vento no outono observadas na torre Colonia Eulacio (linha azul) e simuladas pelas distintas parametrizações utilizadas no modelo WRF-ARW (linha vermelha) para a altura de 101.8 metros

\section{CONCLUSÃO}

Analisando-se todas as parametrizações físicas utilizadas no modelo WRF-ARW percebeu-se que para a altura de 10.1 metros, a configuração com Mellor-Yamada-Janjic para camada limite planetária e Janjic Eta para camada limite superficial, obteve a melhor representatividade do ciclo diário, conseguindo identificar um aumento da velocidade média do vento para períodos diurnos e um decréscimo da velocidade média para períodos noturnos, para todas as estações do ano, o que refletiu o seu baixo valor de erro médio absoluto e alta correlação linear quando comparada as outras parametrizações utilizadas no modelo. Para a altura de 101.8 metros, de modo geral todas as parametrizações físicas utilizadas conseguiram representar de boa maneira o ciclo diário, conseguindo identificar a dimuição da velocidade média do vento nos períodos diurnos e seu aumento na velocidade média para períodos noturnos.

\section{AGRADECIMENTOS}

Agradecemos a Administración Nacional de Usinas y Trasmisiones Eléctricas (UTE) por fornecer os dados que serviram de base ao estudo realizado.

\section{REFERÊNCIAS BIBLIOGRÁFICAS}

1. Skamarock WC, Klemp JB, Dudhia J, Gill DO, Barker DM, Duda MG, Huang X., Wang W, Powers JG. A Description of the Advanced Research WRF Version 3, Boulder: National Center for Atmospheric Research (NCAR), 2008; 113 p.

2. Wyngaard JC. Structure of the Planetary Boundary Layer and Implications for its Modeling. J Applied Meteorol Climatol. 1985 Nov; 24:1131-1142, doi: 10.1175/1520-0450.

3. Kaimal JC, Wyngaard JC, Haugen DA, Coté R, Izumi Y, Caughey SJ, Readings CJ. Turbulence Structure in the Convective Boundary Layer. J Atmospheric Sciences. 1976 Nov; 33:2152-2169. doi: 10.1175/1520-0469.

4. Lucas EA, Gutiérrez AM, Moraes MR, Boezio GC, Cataldo J. Descrição Estatística do Ciclo Diário do Vento nos Primeiros 100 metros da C.L.P. na Localidade de Colonia Eulacio, Uruguai., Ciencia e Natura v38, Ed. Especial IX Workshop Brasileiro de Micrometeorologia. 2016 Jan; 426-434, doi: 0.5902/2179460X20298. 